\title{
Linking of Utah genealogies to outpatient colonoscopy records defines familial aggregation of colorectal polyps and cancer
}

\author{
Therese MF Tuohy ${ }^{1 *}$, Richard Pimentel ${ }^{1}$, Kerry Rowe ${ }^{2}$, Randall Burt ${ }^{1,3}$, Geraldine Mineau ${ }^{1,4}$ \\ From 13th Annual Meeting of the Collaborative Group of the Americas on Inherited Colorectal Cancer \\ Honolulu, Hawaii, USA. 16-17 October 2009
}

\section{Background}

The Utah Population Database (UPDB) is a shared research resource of the University of Utah. It combines Utah genealogies dating back to the 1700s with data from statewide resources, including a cancer registry, hospital claims, as well as birth and death certificates. A project completed in 2008 linked the UPDB to patient demographic records from Intermountain Healthcare which is a non-profit medical system that serves Utah and Southeast Idaho, providing care to approximately $60 \%$ of the resident population. The availability of large numbers of linked, electronically searchable medical records offers unique opportunities for improved clinical and outcomes research. We address the question of the extent to which colonic adenomas and hyperplastic polyps co-segregate with colon cancer risk in non-syndromic families.

\section{Methods}

A pilot project was initiated to use this new linked research infrastructure by ascertaining the occurrence of colorectal polyps from Intermountain clinical data. Deidentified medical information was merged with UPDB family structure and statewide cancer data. These combined data sets provided family structure along with cancer histories to investigate familial aggregation. Cox Regression Analysis was used to assess the relative risk of (a) polyp development and (b) colon cancer for first-, second-and third-degree relatives, by polyp type. Custom kinship analysis tools allowed determination of the excess polyp and cancer risk observed in the kindreds of each case, and differentiated high-risk from population-risk families.

\footnotetext{
* Correspondence: therese.tuohy@hci.utah.edu

${ }^{1}$ Huntsman Cancer Institute, University of Utah, Salt Lake City, Utah 84112 , USA
}

\section{Results}

The queries captured data from over 70,000 positive outpatient colonoscopy procedure and pathology reports from over 58,000 de-identified individuals examined between 1995 and 2009. The queries collected data on age, gender, polyp type, number, size, pathology and were verified by manual review of a randomly selected sample of 200 cases. We identified high-risk kindreds dating to founders born in the late 1700 s, with high relative risks for polyp/cancer. Analysis of discrepancies between excess polyp and excess cancer risks within families allowed us to address the possibility that some cancers may arise in the absence of significant excess polyp risk.

\section{Conclusion}

The combination of a large, electronically searchable medical database (and associated tissue specimens) with a linked genealogical resource offers a powerful platform for the development of hypothesis-driven research.

\section{Acknowledgement}

Funded by the Utah Population Database, and by the Huntsman Cancer Foundation.

\section{Author details}

${ }^{1}$ Huntsman Cancer Institute, University of Utah, Salt Lake City, Utah 84112 , USA. 'Department of Oncology, Intermountain Healthcare, Salt Lake City, Utah, USA. ${ }^{3}$ Department of Medicine, University of Utah, Salt Lake City, Utah 84112, USA. ${ }^{4}$ Department of Oncological Sciences, University of Utah, Salt Lake City, Utah 84112, USA.

Published: 25 May 2010

\section{doi:10.1186/1897-4287-8-S1-P24}

Cite this article as: Tuohy et al:: Linking of Utah genealogies to outpatient colonoscopy records defines familial aggregation of colorectal polyps and cancer. Hereditary Cancer in Clinical Practice 2010 8(Suppl 1):P24. 